\title{
Experimental comparison of control strategies for trajectory tracking for mobile robots
}

\section{Linda Capito*, Pablo Proaño, Oscar Camacho and Andrés Rosales}

Facultad de Ingeniería Eléctrica y Electrónica, Escuela Politécnica Nacional, EPN, Quito, 170517, Ecuador

Email: linda.capito@epn.edu.ec

Email: pablo.proano@epn.edu.ec

Email: ocamacho@ula.ve

Email: oscar.camacho@epn.edu.ec

Email: andres.rosales@epn.edu.ec

*Corresponding author

\section{Gustavo Scaglia}

Instituto de Automática,

Departamento de Ingeniería Química,

Universidad Nacional de San Juan,

San Juan, Argentina

Email: gscaglia@unsj.edu.ar

\begin{abstract}
The purpose of this paper is to implement, test and compare the performance of different control strategies for tracking trajectory for mobile robots. The control strategies used are based on linear algebra, PID controller and on a sliding mode controller. Each control scheme is developed taking into consideration the model of the robot. The linear algebra approaches take into account the complete kinematic model of the robot; and the PID and the sliding mode controller use a reduced order model, which is obtained considering the mobile robot platform as a black-box. All the controllers are tested and compared, firstly by simulations and then, by using a Pioneer 3DX robot in field experiments.
\end{abstract}

Keywords: sliding mode control; SMC; mobile robot; trajectory tracking; linear algebra; controller; algorithm; comparison; control.

Reference to this paper should be made as follows: Capito, L., Proaño, P., Camacho, O., Rosales, A. and Scaglia, G. (2016) 'Experimental comparison of control strategies for trajectory tracking for mobile robots', Int. J. Automation and Control, Vol. 10, No. 3, pp.308-327.

Biographical notes: Linda Capito received his Bachelor in Electronic and Automation Engineering from Escuela Politécnica Nacional (EPN), Quito, Ecuador in December 2015. Her current research interests include automatic control, microcontrollers, control systems and industrial instrumentation. 
Pablo Proaño received his Bachelor in Electronical and Automation Engineering from Escuela Politécnica Nacional (EPN), Quito, Ecuador in December 2015. His current research interests include robotics, microcontrollers and industrial programming.

Oscar Camacho received his BSc in EE from Universidad de Los Andes (ULA), Venezuela in 1984, MSc in Control Engineering from ULA in 1992 and $\mathrm{ME}$ and $\mathrm{PhD}$ in Chemical Engineering from University of South Florida, Tampa, Fl. in 1994 and 1996, respectively. He developed post-doctoral activities at USF in 2001. He has held teaching and researching in ULA, USF and Escuela Politécnica Nacional (EPN) in Ecuador. His current research interests include sliding mode control, long delay systems, and chemical process control. He has authored more than 100 publications in journal and conference proceedings.

Andrés Rosales is a Professor and Research and Social Projection Head at Escuela Politécnica Nacional, Quito. He received his Bachelor in Electronical and Automation Engineering from Escuela Politécnica Nacional (EPN), Quito, Ecuador. He graduated as a $\mathrm{PhD}$ in Control Systems Engineering at Universidad Nacional de San Juan, San Juan, Argentina. He made a research residency at the University of Hannover, Germany, as a DAAD Scholar. His research interests include control systems, education, and industrial processes.

Gustavo Scaglia is a Professor at Universidad Nacional de San Juan, San Juan, Argentina. He received his MSc in Control Systems in 2003 and in 2006, he graduated as a PhD in Control Systems at Universidad Nacional de San Juan. Currently, he develops investigation and education projects at the Automation Institute and the Chemistry Engineering Department at Universidad Nacional de San Juan.

\section{Introduction}

There are an emergent amount of control research on mobile robotics due to the inherent nonlinearity in dynamics and also because they are very useful in industrial applications, in military actions, in challenging access or dangerous areas and for domestic and entertainment activities (Scaglia et al., 2009, 2010; Rosales et al., 2009b, 2010). In practical situations, there are many difficult problems in controlling mobile robots because of the inevitable uncertainties (Toibero et al., 2009; Roth and Batavia, 2012).

The use of trajectory tracking, which is related with the design of control laws that force the robot to reach and follow a time parameterised reference, is justified in structured workspaces as well as in partially structured workspaces, where unexpected obstacles can be found during the navigation. In the first case, the desired trajectory can be set from a global trajectory planner (Rico et al., 2001). In the second case, the algorithms used to avoid obstacles usually re-plan the trajectory in order to avoid a collision, generating a new reference trajectory from this point (Dong-Shu and Hua-Fang, 2011). In general, the objective is to find the control actions that make the mobile robot reach Cartesian position $(x, y)$ with a pre-established orientation $\theta$ in each sampling period. These combined actions result in tracking the desired trajectory of the mobile robot. The actual challenge is to design controllers that can be easily implemented and 
can react appropriately to different kinds of external disturbances, setting of tracking accuracy and robustness (Furat and Eker, 2012; Auat and Scaglia, 2013).

Several control strategies have been proposed for tracking trajectory, some of which are based on either the kinematic or the dynamic models of the mobile robot. The considered model used for this purpose can be both the kinematic model (Hedjar et al., 2005; Kanayama and Kimura, 1990; Kühne et al., 2005; Lee et al., 1999) as well as the dynamic model (Brennan and Alleyine, 2002; Dong and Kuhnert, 2005; Shuli, 2005; Yang and Kim, 1999; Zhang et al., 1998), which use a variety of approximations to obtain the controller, such as the Lyapunov method (Kanayama and Kimura, 1990), fuzzy logic (Lee et al., 1999) or predictive control (Kühne et al., 2005).

Scaglia et al. (2009) presented a control methodology based on the application of linear algebra (LA) for trajectory tracking, where the control actions are obtained by solving a system of linear equations. In order to get this objective, only two control variables are available: the linear velocity $(v)$ and the angular velocity $(\omega)$ of the robot.

By other side, sliding mode control (SMC) is a robust and simple procedure that allows synthesising controllers for linear and nonlinear processes (Utkin, 1977). The main advantages of using SMC are robustness to parameter uncertainty, insensitivity to load disturbance and fast dynamics response. Generally, the design of this controller depends completely on the process model, and the numbers of tuning parameters are in proportion to the model order (Slotine and Li, 1991). The major drawback of SMC is the so called chattering phenomenon. Various approaches of SMC have been proposed to control mobile robots (Jung et al., 2007; Solea et al., 2009; Yue et al., 2011; El'Youssef et al., 2010; Proaño et al., 2015).

This work shows a different approach of SMC for trajectory tracking for mobile robots. The SMC used in this work was developed by Camacho and Smith (2000) for chemical processes and it is based on easy concepts, and there is no need of complex calculations, with low computational cost to achieve the control signal.

The aim of this paper is to make a comparison of different control strategies for tracking trajectory for mobile robots. The control strategies used are two LA controllers, a PID controller and a SMC controller. Each control scheme is developed taking into account the model of the robot. The LA approaches take into account the complete kinematic model of the robot, and the PID and the SMC controllers use a reduced order model of the robot. All the controllers are tested using a Pioneer 3DX robot.

This paper is structured as follows. Section 2 presents the kinematic model of the mobile robot. Section 3 presents the fundamentals and formulation of the proposed controllers. In Section 4, the controllers' synthesis is outlined. In Section 5, simulation, experimental results and their discussion are presented. Finally, Section 6 contains the conclusions.

\section{Process model}

This section describes briefly the process model to be used in the designing of different kind of controllers that will be used and compared in the tracking trajectory of the robot Pioneer 3DX.The model of this robotic platform is depicted in Figure 1. The complete kinematic model is described by equation (1). 
Figure 1 Nonholonomic unicycle robot model (see online version for colours)

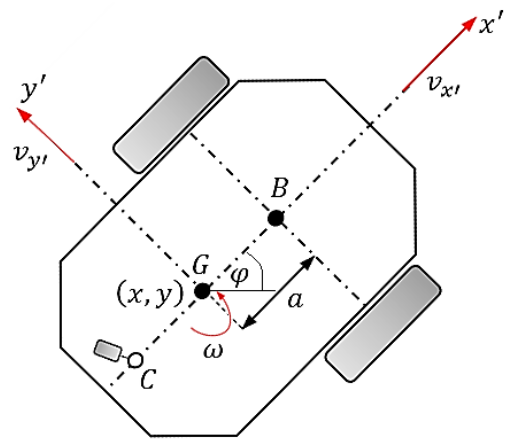

Source: Rosales et al. (2009a)

The position of the robot for analysis is defined by the point $(x, y)$, which locates in this case in the point $G$ (gravity centre). $G$ is a certain distance $a$ from $B$, which is the centre of the line that connects the wheels. $v_{x}^{\prime}$ and $v_{y}^{\prime}$ are the speeds of the centre of mass (longitudinal and lateral), $\omega$ is the angular speed of the robot, $v$ is the linear speed and $\varphi$ is the orientation angle.

$$
\left[\begin{array}{l}
\dot{x} \\
\dot{y} \\
\varphi
\end{array}\right]=\left[\begin{array}{cc}
\cos (\varphi) & -a \sin (\varphi) \\
\sin (\varphi) & a \cos (\varphi) \\
0 & 1
\end{array}\right]\left[\begin{array}{l}
v \\
\omega
\end{array}\right]
$$

The objective of the controllers is to track a reference trajectory that consists of two dimensions to follow, and that are generated according to previously specifications selected by the user, which will be called reference trajectories.

\section{Controllers basic concepts}

In this part, a brief description of the SMC and the two approaches based on LA are presented. The idea is to show basic concepts useful in their application.

\subsection{SMC from a reduced order model of the robot.}

The use of the complete model of the robot for the SMC synthesis becomes in a complex controller. Besides, and considering that there are other factors that influence the performance of the platform (such as worn or old parts or temperature), it is not always possible to use the exact model.

For this reason, the robot is considered as a black box (Figure 2), where only the input (linear speed $v$ and angular speed $\omega$ ) and output (odometry data $x, y$ and $\varphi$ ) are known. Using an identification procedure, the obtained model represents a general one, therefore the resulting controller will be applicable for any other platform with similar behaviour. 
Figure 2 Black box model of a mobile robot

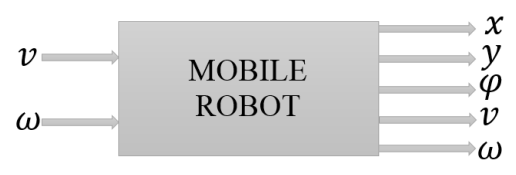

This proposal allows obtaining a reduced order model for designing purposes. Hence, the job of the controller consists on receiving the data from the linear and angular speeds and generates the control signals to reach the speed references and thus the position references, satisfying the desired trajectory tracking.

Figure 3 shows the platform responses when a step signal is applied to the linear and angular speeds, the responses look like as first order plus dead time (FOPDT) models.

Figure 3 Linear and angular speeds step responses (see online version for colours)
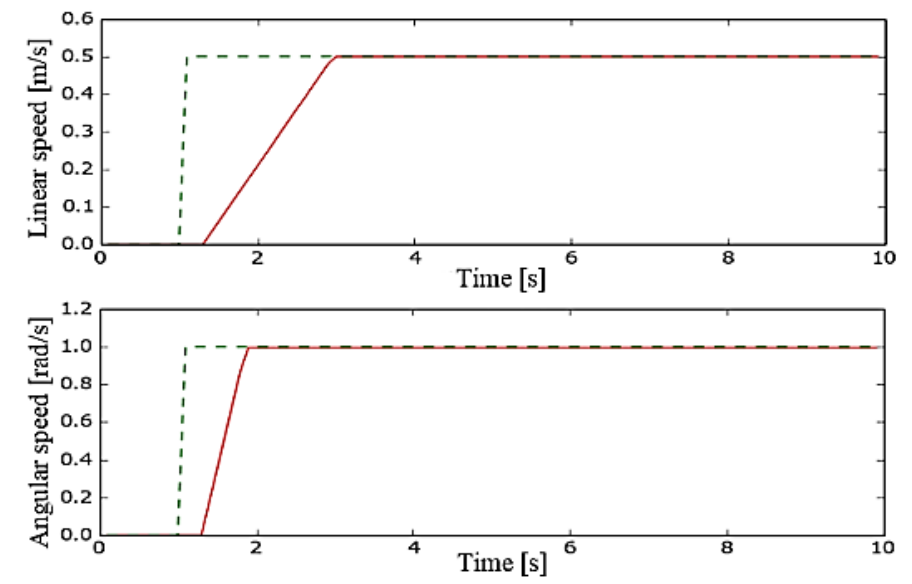

The general form of an FOPDT model can be written as in (2).

$$
\frac{\chi(s)}{U(s)}=\frac{K e^{-t_{0} s}}{\tau s+1}
$$

where
$\chi(s) \quad$ is the process output
$U(s)$ is the controller output
$\tau \quad$ is the time constant of the system
$K \quad$ is the steady state gain of the process
$t_{0} \quad$ is the time delay (deadtime).

The original and the approximated responses are depicted in Figure 4. 
Figure 4 Original and approximation responses (see online version for colours)
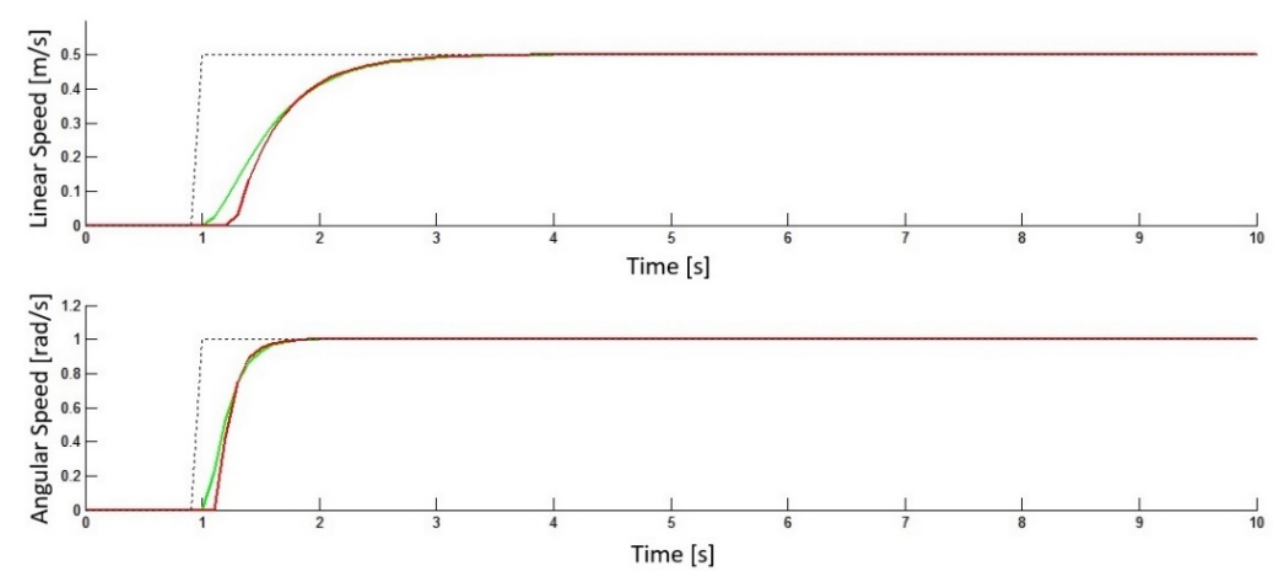

Following the procedure as was presented (Camacho, 1996), the sliding surface can be structured as a PID controller (3).

$$
s(t)=\frac{d e(t)}{d t}+\lambda_{1} e(t)+\lambda_{0} \int_{o}^{t} e(t)
$$

And the SMC can be easily implemented in discrete time by using (Pérez de la Parte, 2005):

$$
U=\frac{\chi(m)}{K}+\frac{\tau_{0} \tau}{K} \lambda_{0}(R(m)-\chi(m))+K_{D} \frac{s(m)}{|s(m)|+\delta}
$$

The tuning parameters (Camacho, 1996), for the continuous part are:

$$
\begin{aligned}
& \lambda_{1}=\frac{\tau+t_{0}}{t_{0} \tau} \\
& \lambda_{0} \leq \frac{\lambda_{1}^{2}}{4}
\end{aligned}
$$

and for the discontinuous part, the parameters are:

$$
\begin{aligned}
& K_{D}=\frac{0.51}{|K|}\left[\frac{\tau}{t_{0}}\right]^{0.76} \\
& \delta=0.68+0.12\left(\begin{array}{lll}
K & K_{D} & \lambda_{1}
\end{array}\right)
\end{aligned}
$$

The characteristic process parameters are obtained from the reaction curve method (Smith and Corripio, 2006). 


\subsection{LA controllers}

The idea behind this approach consists in calculating the optimal control action (Strang, 1980), which allows the robot, to go from the actual to the desired state (Scaglia et al., 2009; Capito and Proaño, 2015).

To apply the method, two approximations are used; these are Euler and trapezoidal approximations. In each case, the kinematic model of robot is substituted in the algebraic expressions and therefore, the controller action calculated.

\subsubsection{For the Euler approximation}

$$
\begin{aligned}
& {\left[\begin{array}{l}
x_{m+1} \\
y_{m+1} \\
\varphi_{m+1}
\end{array}\right]=\left[\begin{array}{l}
x_{m} \\
y_{m} \\
\varphi_{m}
\end{array}\right]=T_{0}\left[\begin{array}{cc}
\cos (\varphi) & -a \sin (\varphi) \\
\sin (\varphi) & a \cos (\varphi) \\
0 & 1
\end{array}\right]\left[\begin{array}{l}
v_{m} \\
\omega_{m}
\end{array}\right]} \\
& T_{0}\left[\begin{array}{cc}
\cos (\varphi) & -a \sin (\varphi) \\
\sin (\varphi) & a \cos (\varphi) \\
0 & 1
\end{array}\right]\left[\begin{array}{l}
v_{m} \\
\omega_{m}
\end{array}\right]=\left[\begin{array}{l}
x_{m+1}-x_{m} \\
y_{m+1}-y_{m} \\
\varphi_{m+1}-\varphi_{m}
\end{array}\right] \\
& \frac{1}{T_{0}}\left[\begin{array}{c}
\Delta x_{m} \\
\Delta y_{m} \\
\Delta \varphi_{m}
\end{array}\right]=\left[\begin{array}{cc}
\cos (\varphi) & -a \sin (\varphi) \\
\sin (\varphi) & a \cos (\varphi) \\
0 & 1
\end{array}\right]\left[\begin{array}{l}
v_{m} \\
\omega_{m}
\end{array}\right]
\end{aligned}
$$

Therefore, it can be represented as:

$$
A \boldsymbol{u}=\boldsymbol{b}
$$

where

$$
\boldsymbol{u}=\left[\begin{array}{c}
v_{m} \\
\omega_{m}
\end{array}\right]
$$

The speeds $v_{m}$ and $\omega_{m}$ need to be determined. When having an inconsistent system, the least square method (Strang, 1980) is used to satisfy the equation (12) and find the expression for $\boldsymbol{u}$.

$$
A^{T} A \boldsymbol{u}=A^{T} \boldsymbol{b}
$$

Applying this method, the expressions for the desired $v_{m}$ and $\omega_{m}$ are found:

$$
\begin{aligned}
& v_{m}=\frac{1}{T_{0}}\left(\Delta x_{m} \cos \left(\varphi_{m}\right)+\Delta y_{m} \sin \left(\varphi_{m}\right)\right) \\
& \omega_{d m}=\frac{1}{T_{0}\left(a^{2}+1\right)}\left(-a^{2} \Delta x_{m} \sin \left(\varphi_{m}\right)+a \Delta y_{m} \cos \left(\varphi_{m}\right)+\Delta \varphi_{m}\right)
\end{aligned}
$$




\subsubsection{For the trapezoidal approximation}

Following the procedure presented in Section 3.2.1, we have:

$$
\begin{aligned}
& {\left[\begin{array}{l}
x_{m+1} \\
y_{m+1} \\
\varphi_{m+1}
\end{array}\right]=\left[\begin{array}{l}
x_{m} \\
y_{m} \\
\varphi_{m}
\end{array}\right]+\frac{T_{0}}{2}\left(\left[\begin{array}{c}
v_{m} \cos \left(\varphi_{m}\right)-a \omega_{m} \sin \left(\varphi_{m}\right) \\
v_{m} \sin \left(\varphi_{m}\right)+a \omega_{m} \cos \left(\varphi_{m}\right) \\
\omega_{m}
\end{array}\right]\right.} \\
& \left.+\left[\begin{array}{cc}
\cos \left(\varphi_{m+1}\right) & -a \sin \left(\varphi_{m+1}\right) \\
\sin \left(\varphi_{m+1}\right) & a \cos \left(\varphi_{m+1}\right) \\
0 & 1
\end{array}\right]\left[\begin{array}{c}
v_{m+1} \\
\omega_{m+1}
\end{array}\right]\right) \\
& {\left[\begin{array}{c}
\Delta x_{m} \\
\Delta y_{m} \\
\Delta \varphi_{m}
\end{array}\right]=\frac{2}{T_{0}}-\left[\begin{array}{c}
v_{m} \cos \left(\varphi_{m}\right)-a \omega_{m} \sin \left(\varphi_{m}\right) \\
v_{m} \sin \left(\varphi_{m}\right)+a \omega_{m} \cos \left(\varphi_{m}\right) \\
\omega_{m}
\end{array}\right]} \\
& =\left[\begin{array}{cc}
\cos \left(\varphi_{m+1}\right) & -a \sin \left(\varphi_{m+1}\right) \\
\sin \left(\varphi_{m+1}\right) & a \cos \left(\varphi_{m+1}\right) \\
0 & 1
\end{array}\right]\left[\begin{array}{c}
v_{m+1} \\
\omega_{m+1}
\end{array}\right] \\
& {\left[\begin{array}{cc}
\cos \left(\varphi_{m+1}\right) & -a \sin \left(\varphi_{m+1}\right) \\
\sin \left(\varphi_{m+1}\right) & a \cos \left(\varphi_{m+1}\right) \\
0 & 1
\end{array}\right]\left[\begin{array}{c}
v_{m+1} \\
\omega_{m+1}
\end{array}\right]=\left[\begin{array}{c}
\frac{2}{T_{0}} \Delta x_{m}-v_{m} \cos \left(\varphi_{m}\right)+a \omega_{m} \sin \left(\varphi_{m}\right) \\
\frac{2}{T_{0}} \Delta y_{m}-v_{m} \sin \left(\varphi_{m}\right)-a \omega_{m} \cos \left(\varphi_{m}\right) \\
\frac{2}{T_{0}} \Delta \varphi_{m}-\omega_{m}
\end{array}\right]}
\end{aligned}
$$

In a similar way, as it was presented for the Euler approximation

$$
A \boldsymbol{u}=\boldsymbol{b}
$$

where

$$
\boldsymbol{u}=\left[\begin{array}{c}
v_{m+1} \\
\omega_{m+1}
\end{array}\right]
$$

To find $v_{m}$ and $\omega_{m}$ we use again the least square method (Strang, 1980) to solve this inconsistent system (20) and find the expression for $\boldsymbol{u}$.

$$
A^{T} A \boldsymbol{u}=A^{T} \boldsymbol{b}
$$

Applying this method the expressions for the desired $v_{m}$ and $\omega_{m}$ are now found for this case:

$$
v_{m+1}=\frac{2}{T_{0}} \varepsilon+\epsilon
$$


where

$$
\varepsilon=\left(\Delta x_{m} \cos \left(\varphi_{m+1}\right)+\Delta y_{m} \sin \left(\varphi_{m+1}\right)\right)
$$

and

$$
\begin{aligned}
\epsilon & =-v_{m} \cos \left(\varphi_{m+1}-\varphi_{m}\right)+a \omega_{m} \sin \left(\varphi_{m}-\varphi_{m+1}\right) \\
\omega_{m+1} & =\frac{1}{a^{2}+1}(\gamma+\vartheta)
\end{aligned}
$$

where

$$
\gamma=\frac{2}{T_{0}} \Delta \varphi_{m}-\omega_{m}+\frac{2 a}{T_{0}}\left(\Delta y_{m} \cos \left(\varphi_{m+1}\right)-\Delta x_{m} \sin \left(\varphi_{m+1}\right)\right)
$$

and

$$
\vartheta=a v_{m} \sin \left(\varphi_{m+1}-\varphi_{m}\right)-a^{2} \omega_{m} \cos \left(\varphi_{m+1}-\varphi_{m}\right)
$$

\subsubsection{Minimisation of the error}

To minimise the error a vector $\boldsymbol{b}(\boldsymbol{C A})$ should be found, it belongs to the column space of the matrix $A$ and that is the closest to vector $\boldsymbol{b}(28)$ :

$$
A \boldsymbol{u}=\boldsymbol{b}=\boldsymbol{b}(\boldsymbol{C A})+\boldsymbol{b}\left(\boldsymbol{N} \boldsymbol{A}^{T}\right)
$$

The column space of $A$ is the group of vectors in $\mathbb{R}^{m}$ that can be expressed as a linear combination of the $n$ columns of the matrix $A$, and it can be represented as follows.

$$
k_{1} \boldsymbol{v}_{1}+k_{2} \boldsymbol{v}_{2}=\boldsymbol{v}_{3}=\overline{\boldsymbol{b}(\boldsymbol{C A})}
$$

where $\boldsymbol{v}_{1}$ and $\boldsymbol{v}_{2}$ are base vectors of the column space of $A$ and they are perpendicular. The constants $k_{1}$ and $k_{2}$ correspond therefore to the speeds $v_{m}$ and $\omega_{m}$ previously found.

To ensure an exact solution, the $\boldsymbol{b}$ component on the left side of the null space of $A^{T}$ must be zero (Scaglia et al., 2009):

$$
\boldsymbol{b}\left(N \boldsymbol{A}^{\boldsymbol{T}}\right)=\mathbf{0}
$$

Finally (29) and (30) are replaced in (28) in order to obtain the expression that ensures that the minimum error is made. The obtained expression is a restriction for the selection of the angle $\varphi_{e z_{m}}$ which allows guiding the robot every sample time to its next goal, as observed in Figure 5.

Using the obtained value for the angle, it is assured that the solution is exact or has minimum error (Rosales et al., 2009a). 
Figure 5 Robot's orientation with angle (see online version for colours)

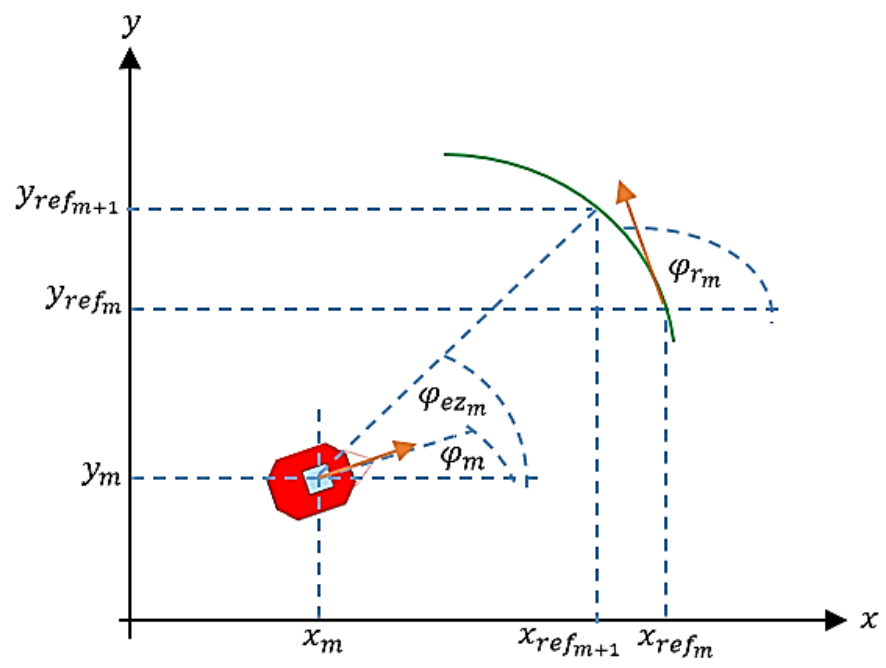

Minimising the error we obtain the following angle restrictions:

- For the Euler approximation

$$
\varphi_{e z_{m}}=\tan ^{-1}\left(\frac{\Delta y_{m}}{\Delta x_{m}}\right)
$$

- For the trapezoidal approximation

$$
\varphi_{e z_{m}}=\tan ^{-1}\left(\frac{\Delta y_{m} \frac{2}{T_{0}}-v_{m} \sin \left(\varphi_{m}\right)}{\Delta x_{m} \frac{2}{T_{0}}-v_{m} \cos \left(\varphi_{m}\right)}\right)
$$

\subsubsection{Considerations to make the error tend to zero stably}

Equation (33) is used to guarantee that the error will decrease smoothly as the time elapses. This is necessary to avoid that the system reaches the reference abruptly, which could cause instabilities (Rosales et al., 2009a).

$$
\begin{aligned}
& x_{m+1}=x_{r e f_{m+1}}-k_{x}\left(x_{r e f_{m}}-x_{m}\right) \\
& 0<k_{x}<1
\end{aligned}
$$

where $k_{x}$ defines the speed with which the error will reach zero, and it must be between zero and one (34). Replacing the position data in (33) and solving, the set of equations for $\Delta x, \Delta y$ and $\Delta \varphi_{e z},(35)$ is obtained. 


$$
\begin{aligned}
\Delta x & =x_{r e f_{m+1}}-x_{m}-k_{x}\left(x_{r e f_{m}}-x_{m}\right) \\
\Delta y & =y_{r e f_{m+1}}-y_{m}-k_{y}\left(y_{r e f_{m}}-y_{m}\right) \\
\Delta \varphi_{e z} & =\Delta \varphi_{e z_{m}}-\varphi_{m}-k_{\varphi}\left(\varphi_{e z_{m}}-\varphi_{m}\right)
\end{aligned}
$$

\section{Experimental results}

In this section, the four controllers are tested and their performance compared firstly by simulations and then in a realistic way. In the simulation case, a double frequency trajectory is used. This test could not be verified by real experimentation due to insufficient physical space. In the realistic experiments, square and circular trajectories are used.

The IAE index (Kealy and O'Dwyer, 2003; Himmelblau, 1972) is used to measure the performance of the controllers; it is defined as in (36).

$$
\overline{I A E}=\frac{\int_{0}^{T}|e(t)| d t}{T}
$$

The controller with the minimum IAE has the best performance.

\subsection{Simulation for a double frequency trajectory}

Figures 6, 7, 8, 9 and 10 illustrate how perform each controller for a double frequency trajectory. Figure 6 is a XY graph, Figure 7 shows the $X$ and $Y$ responses vs. time, Figure 8 is the trajectory error responses vs. time, in Figure 9 linear and angular speeds vs. time are plotted, finally Figure 10 displays the angular positions vs. time.

Figure 6 XY graph (see online version for colours)

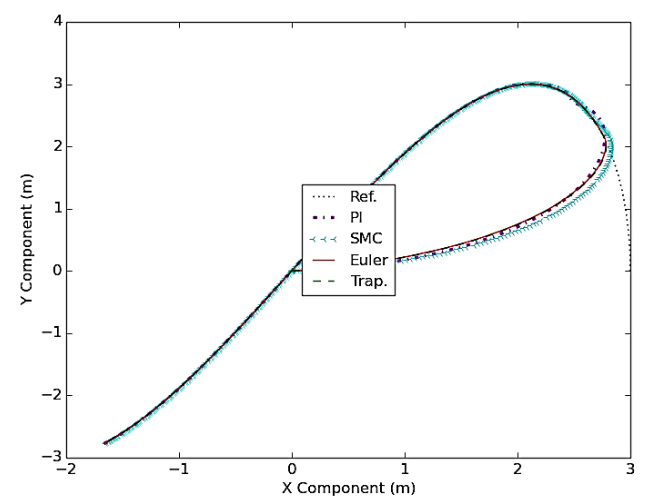


Figure $7 \quad \mathrm{X}$ and $\mathrm{Y}$ trajectory vs. time (see online version for colours)
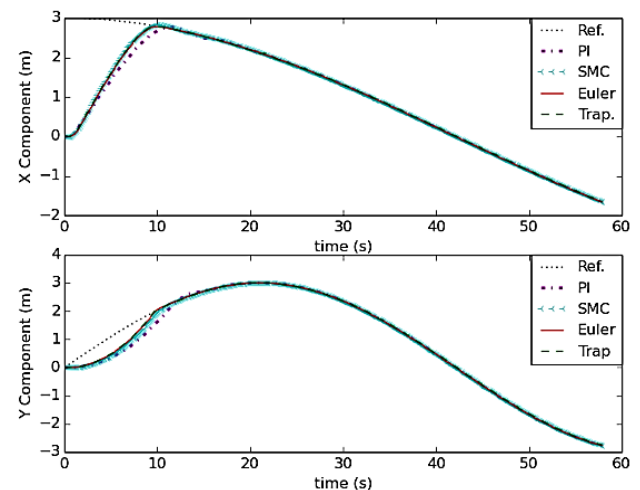

Figure 8 Trajectory error vs. time (see online version for colours)

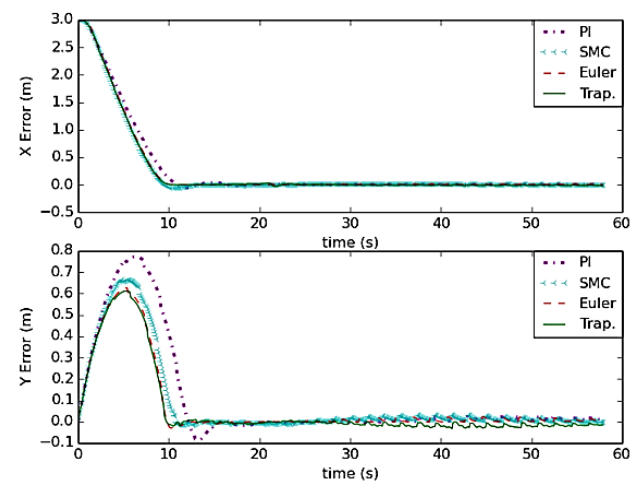

Figure 9 Linear and angular speeds vs. time (see online version for colours)
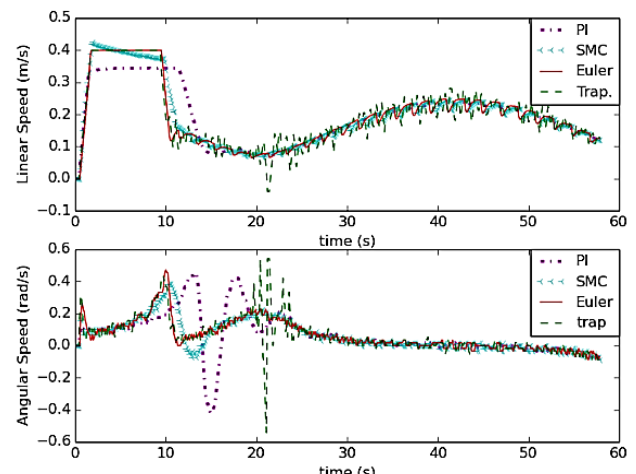
Figure 10 Angular position vs. time (see online version for colours)

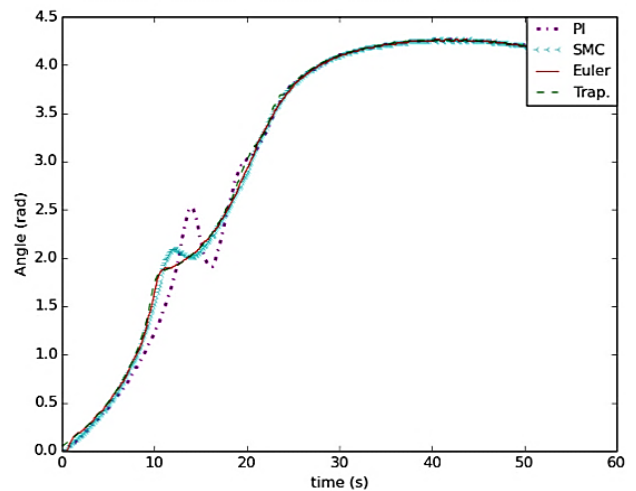

Table 1 IAE comparison - double frequency trajectory

\begin{tabular}{lcccc}
\hline$\infty$ & Euler & Trapezoidal & PI & SMC \\
\hline 250 & ${\text { IAE } * 10^{-3}}^{-3}$ & IAE $* 10^{-3}$ & IAE $* 10^{-3}$ & ${\text { IAE } * 10^{-3}}^{-3}$ \\
$\mathrm{X}$ & 10,183 & 10,685 & 11,290 & 10,343 \\
$\mathrm{Y}$ & 3,236 & 3,952 & 5,080 & 4,008 \\
\hline
\end{tabular}

The first thing to notice is the fast response of the SMC, it is the first controller to reach the desired path. The performance of the LA controllers are practically the same, the Euler approach presents the best results. The PI appears to be the slowest, but its overall performance is very acceptable. In spite that the SMC, uses a reduced order model of the robot, its overall performance is very close to the LA approaches which use a complete model of the robot, and much better than the PI controller.

\subsection{Realistic experiments}

In this part, two different trajectories are used: a square one with each side $=2.5 \mathrm{~m}$ and a circular one with radius $=2 \mathrm{~m}$. Figure 11 shows a picture of the Pioneer 3DX that was used in these experimental tests with the four controllers.

Figure 11 Pioneer 3DX (see online version for colours)

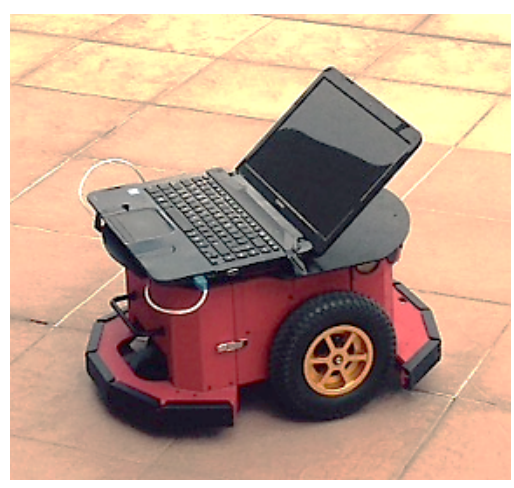




\subsubsection{Square trajectory}

Figure 12 shows the Pioneer 3DX responses for each controller. Figures 13 to 16 depict the same aspects as in the simulation case.

Figure $12 \mathrm{XY}$ graph (see online version for colours)

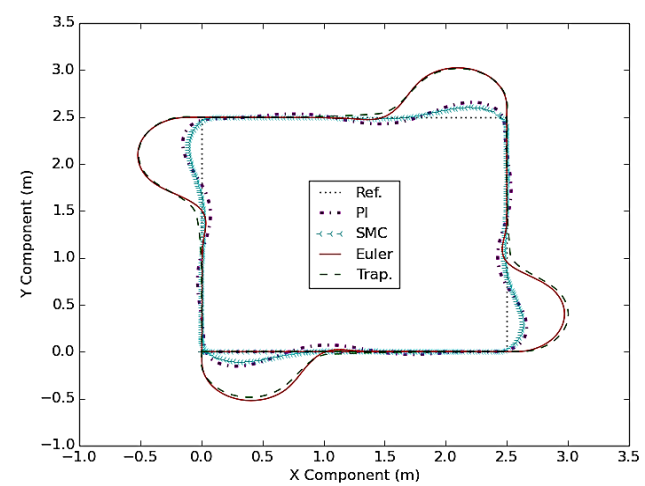

Figure $13 \mathrm{X}$ and $\mathrm{Y}$ trajectory vs. time (see online version for colours)
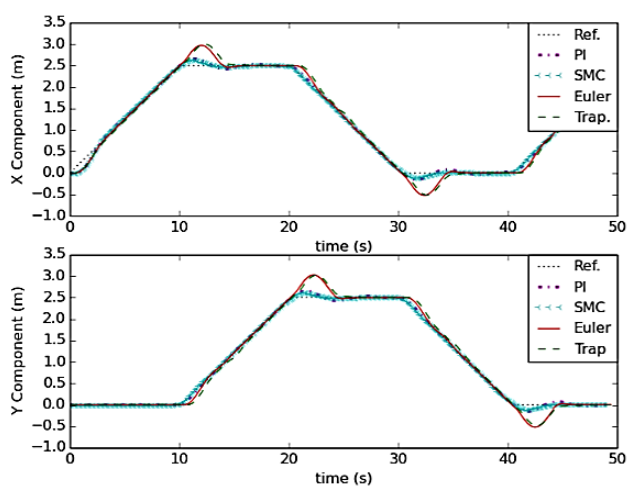

Figure 14 Trajectory error vs. time (see online version for colours)

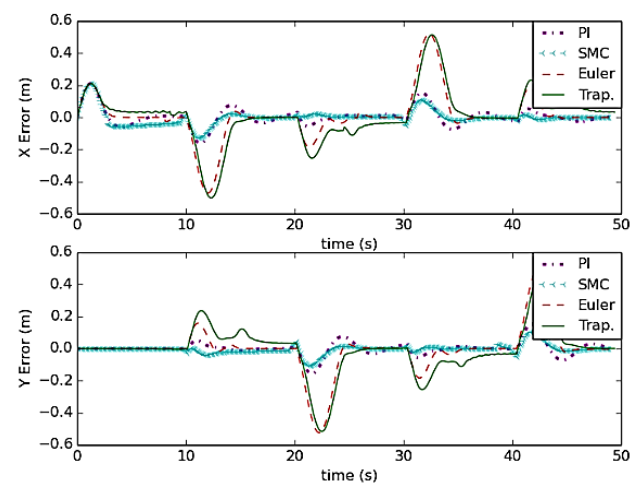


Figure 15 Linear and angular speed vs. time (see online version for colours)

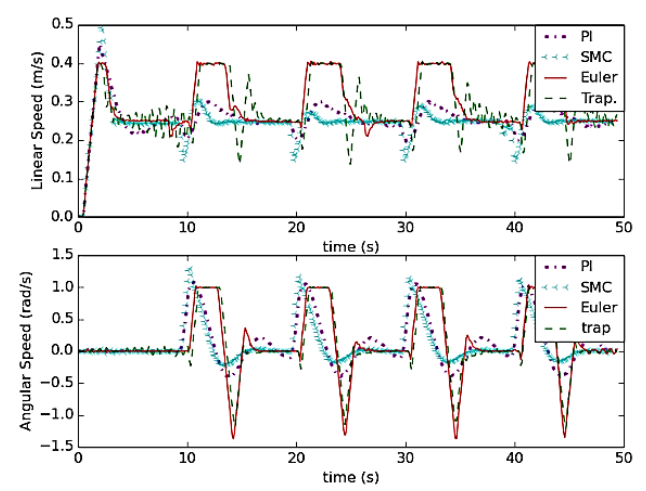

Figure 16 Angular position vs. time (see online version for colours)

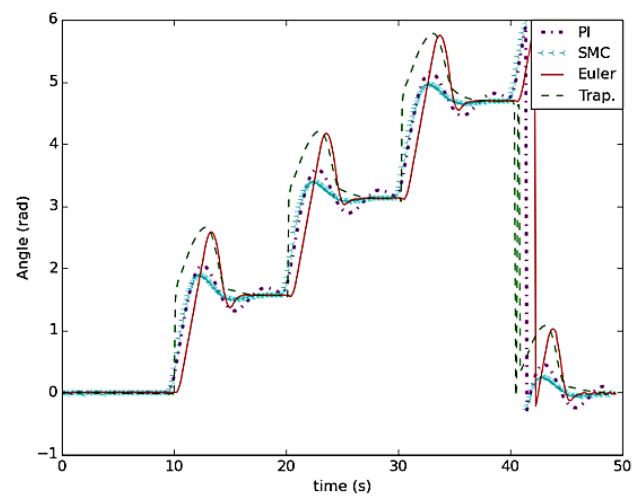

Table 2 IAE comparison - square trajectory

\begin{tabular}{lcccc}
\hline$\square$ & Euler & Trapezoidal & $P I$ & $S M C$ \\
\hline 250 & ${\text { IAE } * 10^{-3}}^{-3}$ & IAE * $10^{-3}$ & IAE * $10^{-3}$ & ${\text { IAE } * 10^{-3}}^{-3}$ \\
$\mathrm{Y}$ & 7,127 & 9,879 & 3,042 & 2,582 \\
$\mathrm{Y}$ & 6,509 & 8,702 & 2,128 & 1,460 \\
\hline
\end{tabular}

According to the results shown in the previous plots and Table 2, it is possible to appreciate that the SMC presents the best reaction when facing abrupt changes, and also the best performance. The PI controller reacts well in this experiment, presenting the second best performance. If the speed increases, the LA controllers take more time to react; this causes bigger overshoots and longer settling times. This happens because of the structure of the controllers by themselves, as the Euler and trapezoid approximation, can anticipate only the next sample time.

\subsubsection{Circular trajectory}

In a similar way as was presented for the square trajectory, Figures 17, 18, 19, 20 and 21 display the same experiments as before. 
Figure $17 \mathrm{XY}$ graph (see online version for colours)

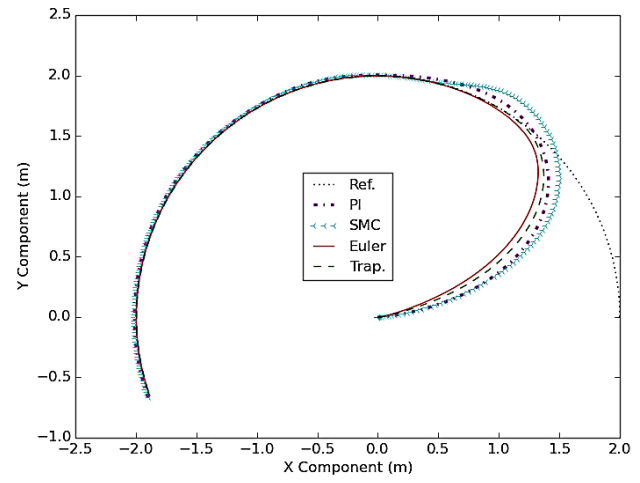

Figure $18 \mathrm{X}$ and $\mathrm{Y}$ trajectory vs. time (see online version for colours)
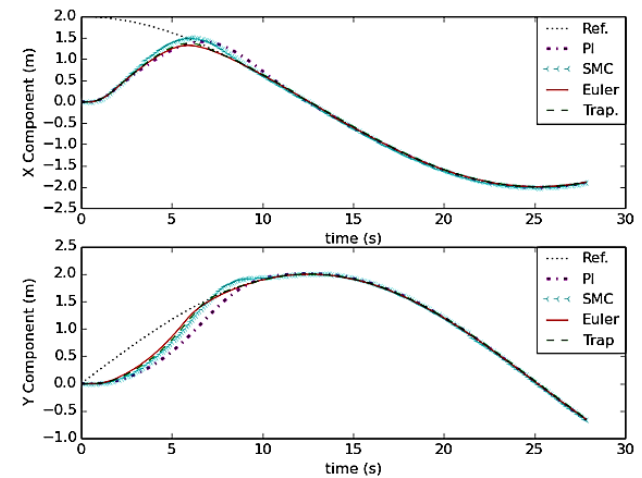

Figure 19 Trajectory error vs. time (see online version for colours)
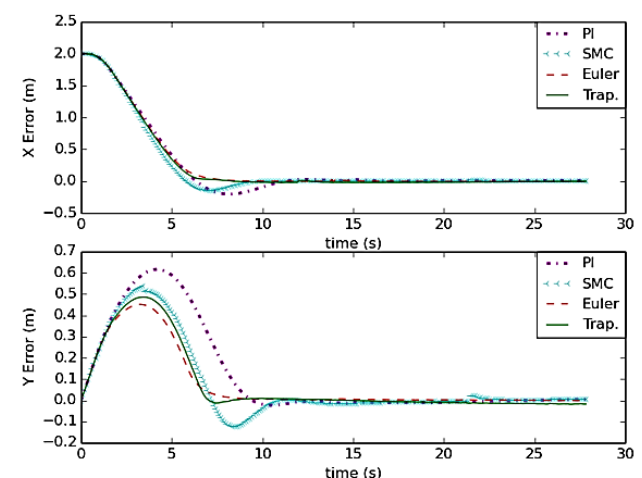
Figure 20 Linear and angular speed vs. time (see online version for colours)

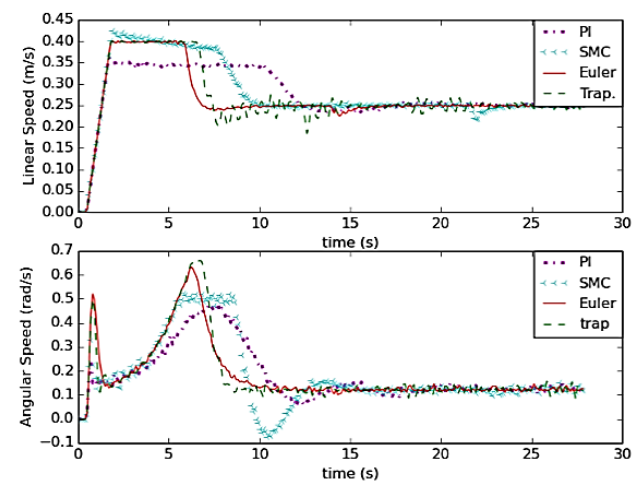

Figure 21 Angular position vs. time (see online version for colours)

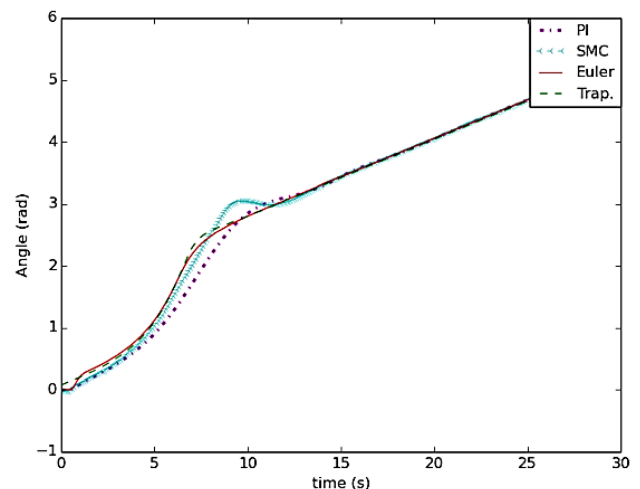

Table 3 IAE comparison - circular trajectory

\begin{tabular}{lcccc}
\hline$O$ & Euler & Trapezoidal & $P I$ & $S M C$ \\
\hline 250 & ${\text { IAE } * 10^{-3}}^{-3}$ & IAE $* 10^{-3}$ & ${\text { IAE } * 10^{-3}}^{*}$ & IAE $^{-3} 10^{-3}$ \\
$\mathrm{X}$ & 8,027 & 7,438 & 8,566 & 7,792 \\
$\mathrm{Y}$ & 3,163 & 2,284 & 4,246 & 3,433 \\
\hline
\end{tabular}

For the circular trajectory the results presented indicate that the trapezoidal approximation has the lowest settling time, and Table 3 provides that the trapezoidal approximation produces the best performance. The SMC presents a good response when reaching its trajectory, however, as the speed increases the overshoot increases too. It can be seen from Table 3 that the SMC presents a very close performance index compared with LA controllers. 


\section{Conclusions}

The controllers developed using LA approximations have shown good performances in general way, the stabilisation times are similar for both controllers. On the other side, the PID and the SMC, from a reduced order model of the robot, produce in general satisfactory results, especially for the square trajectory, where they presented the best responses.

The experimental results, simulations and field tests, indicated that the presented SMC has a good performance, better than the PI controller, and closer to those controllers based on the complete model of the robot.

Moreover, the proposed SMC approach used in this work can be easily implemented since it uses a PID controller as sliding surface and also presents a set of tuning equations based on the characteristic parameters of the process.

\section{Acknowledgements}

The authors would like to thank Escuela Politécnica Nacional - EPN, Ecuador, for the support to conduct this research project. The third author would like to thank PROMETEO Project of SENESCYT, Republic of Ecuador, for its sponsorship for the realisation of this work. The authors would like to thank the Universidad de Las Fuerzas Armadas - ESPE, Ecuador, for providing them a robot Pioneer 3DX to conduct the realistic experimental tests included in this paper.

\section{References}

Auat, F. and Scaglia, G. (2013) 'Trajectory tracking controller design for unmanned vehicles: a new methodology', Journal of Field Robotics, Vol. 31, No. 6, pp.861-887.

Brennan, S. and Alleyne, A. (2002) 'H-infinity vehicle control using nondimensional perturbation measures', American Control Conference, pp.2534-2539.

Camacho, O. (1996) A New Approach to Design and Tune Sliding Mode Controllers for Chemical Processes, $\mathrm{PhD}$ dissertation, University of South Florida, Tampa, Florida.

Camacho, O. and Smith, C. (2000) 'Sliding mode control: an approach to regulate nonlinear chemical processes', ISA Transactions, Vol. 39, No. 2, pp.205-218.

Capito, L. and Proaño, P. (2015) Trajectory Tracking Through Four Control Techniques Using a Robot Pioneer $3 D X$ and the Robotic Operative System ROS, Bachelor thesis, Escuela Politécnica Nacional - EPN, Quito, Ecuador.

Dong, W. and Kuhnert, K. (2005) 'Robust adaptive control of nonholonomic mobile robot with parameter and nonparameter uncertainties', IEEE Transaction on Robotics, Vol. 21, No. 2, pp.261-266.

Dong-Shu, W. and Hua-Fang, Y. (2011) 'Path planning of mobile robot in dynamic environments', Proceedings of the International Conference on Intelligent Control and Information Processing (ICICIP), pp.691-696.

El'Youssef, E.S., Martins, N.A., de Pieri, E.R., Bertol, D.W. and Jungers, M. (2010) 'A wheeled mobile robot trajectory tracking control: 1 st and 2nd order sliding modes applied to a compensated inverse dynamics', 11th Pan-American Congress of Applied Mechanics PACAM XI, Foz do Iguaçu, PR, Brazil. 
Furat, M. and Eker, I. (2012) 'Experimental evaluation of sliding mode control techniques', Çukurova University, Journal of the Faculty of Engineering and Architecture, Vol. 27, No. 1, pp.23-37.

Hedjar, R., Toumi, R. and Boucher, P. (2005) 'Finite horizon nonlinear predictive control by the Taylor approximation', International Journal of Applied Mathematics and Computer Science, Vol. 15, No. 4, pp.527-540.

Himmelblau, D.M. (1972) Applied Nonlinear Programming, McGraw-Hill, New York.

Jung, S., Jeon, P. and Hsia, T. (2007) 'Contour tracking of an unknown planar object by regulating force for mobile robot navigateon', Robotica, Vol. 25, No. 3, pp.297-305.

Kanayama, Y. and Kimura, Y. (1990) 'A stable tracking control method for an autonomous mobile robot', IEEE Robotics and Automation International Conference, Cincinnati, Vol. 1, pp.384-389.

Kealy, T. and O'Dwyer, A. (2003) Analytical ISE Calculation and Optimum Control System Design, IISC, Limerick.

Kühne, F., Gomes, J. and Fetter, W. (2005) 'Mobile robot trajectory tracking using model predictive control', II IEEE LARS, São Luís, pp.1-7.

Lee, T., Leung, F. and Tam, P. (1999) 'Position control for wheeled mobile robots using a fuzzy logic controller', IECON Proceedings, pp.525-528.

Pérez de la Parte, M. (2005) Control Predictivo con Modos Deslizantes, PhD thesis, Universidad de Sevilla, Sevilla, Spain.

Proaño, P., Capito, L., Rosales, A. and Camacho, O. (2015) 'Sliding mode control: implementation like PID for trajectory-tracking for mobile robots', Asia-Pacific Conference on Computer Aided System Engineering (APCASE 2015), Quito, Ecuador, 14-16 July.

Rico, J., Alcalá, I., Gómez-Ortega, J. and Camacho, E. (2001) 'Mobile robot path tracking using PID controller', Control Engineering Practice, Vol. 9, No. 11, pp.1209-1214.

Rosales, A., Scaglia, G. and Mut, V. (2009a) 'Mobile robots navigation in unstructured environments using linear algebra - Navegación de robots móviles en entornos no estructurados utilizando álgebra lineal', Revista Iberoamericana de Automática e Informática, Vol. 6, No. 2, pp.79-88.

Rosales, A., Scaglia, G., Mut, V. and Di Sciascio, F. (2009b) 'Dynamic control and collision avoidance of mobile robots - a linear algebra approach', Robotica, Vol. 27, No. 7, pp.981-997.

Rosales, A., Scaglia, G., Mut, V. and Di Sciascio, F. (2010) 'Formation control and trajectory tracking of mobile robotic systems - a linear algebra approach', Robotica, Vol. 29, No. 3, pp.335-349.

Roth, S. and Batavia, P. (2012) 'Evaluating path tracker performance for outdoor mobile robots', Automation Technology for Off-Road Equipment [online] http://www.ri.cmu.edu/pub_files/ pub4/roth_stephan_a_2002_1/roth_stephan_a_2002_1.pdf (accessed 20 July 2015).

Scaglia, G., Quintero, L., Mut, V. and Di Sciascio, F. (2009) 'Numerical methods based controller design for mobile robots', Robotica, Vol. 27, No. 2, pp.269-279.

Scaglia, G., Rosales, A., Quintero, L., Mut, V. and Agarwal, R. (2010) 'A linear interpolation based controller design for trajectory tracking of mobile robots', Control Engineering Practice, Vol. 18, No. 3, pp.318-329.

Shuli, S. (2005) 'Designing approach on trajectory tracking control of mobile robots', Robotics and Computer Integrated Manufacturing, Vol. 21, No. 1, pp.81-85.

Slotine, J. and Li, W. (1991) Applied Nonlinear Control, Prentice-Hall, New Jersey, USA.

Smith, C. and Corripio, A. (2006) Principles and Practice of Automatic Process Control, 3rd ed., John Wiley \& Sons, Inc., New Jersey, USA.

Solea, R., Filipescu, A. and Nunes, U. (2009) 'Sliding-mode control for trajectory-tracking of a wheeled mobile robot in presence of uncertainties', Asian Control Conference 2009, China.

Strang, G. (1980) Linear Algebra and Its Applications, Academic Press, New York. 
Toibero, J., Roberti, F. and Carelli, R. (2009) 'Stable contour-following control of wheeled mobile robots', Robotica, Vol. 27, No. 1, pp.1-12.

Utkin, V.I. (1977) 'Variable structure systems with sliding modes', IEEE Transactions on Automatic Control, Vol. 22, No. 2, pp.212-222.

Yang, J. and Kim, H. (1999) 'Sliding mode control for trajectory tracking of nonholonomic wheeled mobile robots', IEEE Transactions on Robotics and Automation, Vol. 15, No. 3, pp.579-588.

Yue, M., Sun, W. and Hu, P. (2011) 'Sliding mode robust control for two-wheeled mobile robot with lower center of gravity', International Journal of Innovative Computing, Information and Control ICIC, Vol. 7, No. 2, pp.637-646.

Zhang, Y., Hong, D., Chung, J. and Velinsky, S. (1998) 'Dynamic model based robust tracking control of a differentially steered wheeled mobile robot', American Control Conference 1998, Proceedings of the 1998, Vol. 2, pp.850-855. 